\title{
Efecto de las giberelinas en la propagación de tomate (Solanum lycopersicum L.) bajo diferentes sustratos enriquecidos con fertilizante
}

\section{Effect of gibberellins on the propagation of the tomato (Solanum lycopersicum L.) with different substrates enriched with fertilizer}

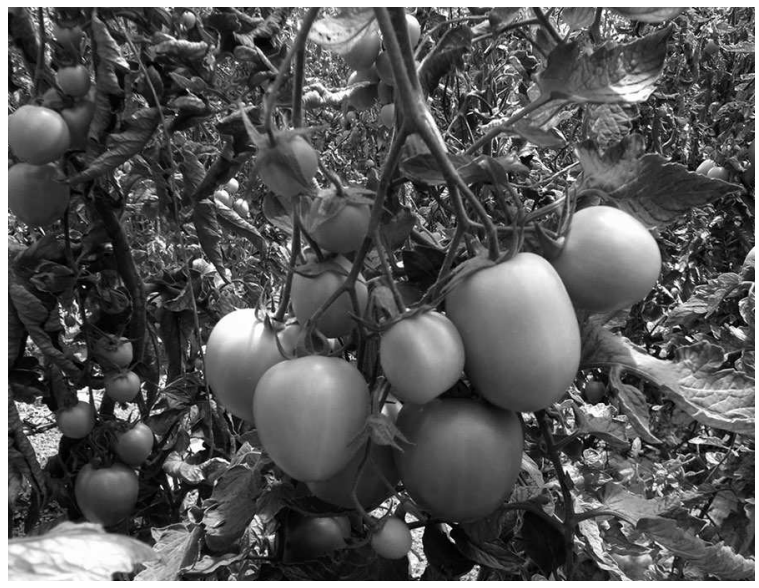

ANA LUCÍA FRAILE-ROBAYO'

JAVIER GIOVANNI ÁLVAREZ-HERRERA2,3

YULI ALEXANDRA DEAQUIZ-OYOLA²

Tomate híbrido Calima con 60 días después de trasplante.

Foto: A.L. Fraile-Robayo

\section{RESUMEN}

En el cultivo de tomate la realización de semilleros o almácigos genera un alto costo de producción, por tal razón se buscan alternativas que brinden características óptimas de germinación y crecimiento de las plántulas, que a la vez sean viables y económicamente fáciles de adquirir. En este estudio se evaluó la respuesta de semillas de tomate híbrido Calima a cinco concentraciones de ácido giberélico (0, 200, 400, 600, $800 \mathrm{mg}$ $\mathrm{L}^{-1}$ ) sembradas en cascarilla de arroz quemada (CAQ) enriquecida con diferentes dosis de nitrógeno, fósforo y potasio (90-75-180, 75-60-165, 60-45-150 y 45-30-135 $\mathrm{mg} \mathrm{L}^{-1}$ ) respectivamente, distribuidas en 12, 16 y 20 días después de la siembra. Se utilizó un diseño completamente aleatorizado, con 25 tratamientos y cuatro repeticiones, cada unidad experimental con 20 semillas. La concentración de $400 \mathrm{mg} \mathrm{L}^{-1}$ de $\mathrm{GA}_{3}$ incidió favorablemente en el tiempo medio de germinación y la velocidad media de germinación, sin embargo no tuvo ningún efecto en el desarrollo de la plántula. El sustrato CAQ más la aplicación de fertilizante presentó plántulas con un desarrollo inferior respecto a la turba, mientras que la mezcla de turba más la aplicación de $400 \mathrm{mg} \mathrm{L}^{-1}$ de $\mathrm{GA}_{3}$ presentó plántulas con mayor crecimiento lo cual se expresó en la altura de plántula $(10 \mathrm{~cm})$, un diámetro de tallo de 3,1 mm y una masa fresca de $0,64 \mathrm{~g}$.

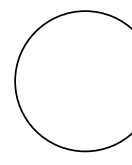

Palabras clave adicionales: germinación, plántula, cascarilla de arroz quemada, turba.

\section{Químicos Oma, Bogotá (Colombia).}

2 Facultad de Ciencias Agropecuarias, Grupo de Investigaciones Agrícolas, Universidad Pedagógica y Tecnológica de Colombia, Tunja (Colombia).

3 Autor para correspondencia. jgalvarezh@gmail.com 


\section{ABSTRACT}

In the tomato, the use of seedbeds causes a high production cost, therefore alternatives are being sought that provide optimal characteristics of germination and seedling growth that are both economically viable and available. This study evaluated the response of Calima hybrid tomato seeds treated before sowing with five concentrations of gibberellic acid $\left(0,200,400,600,800 \mathrm{mg} \mathrm{L}^{-1}\right)$, planted in burned rice husks (CAO) and enriched with different doses of nitrogen, phosphorous and potassium (90-75-180, 45-30-135 and 75-60-165, $60-45-150 \mathrm{mg} \mathrm{L}^{-1}$ ) respectively, at 12,16 and 20 days after planting; and compared with peat moss. We used a completely randomized design with 25 treatments and four replications; each experimental unit had 20 seeds. The concentration of $400 \mathrm{mg} \mathrm{L}^{-1}$ positively affected the mean germination time and the speed of germination, but had no effect on the development of the seedlings. The CAO substrate enriched with fertilizer presented seedlings with lower development as compared to peat moss, while the mixture of peat moss plus $400 \mathrm{mg}$ $\mathrm{L}^{-1} \mathrm{GA}_{3}$ had the fastest growing seedlings which expressed a height of $10 \mathrm{~cm}$, a stem diameter of $3.1 \mathrm{~mm}$ and $0.64 \mathrm{~g}$ of fresh biomass.

Additional key words: germination, seedling, burned rice husks, peat.

Fecha de recepción: 10-04-2012

Aprobada para publicación: 29-05-2012
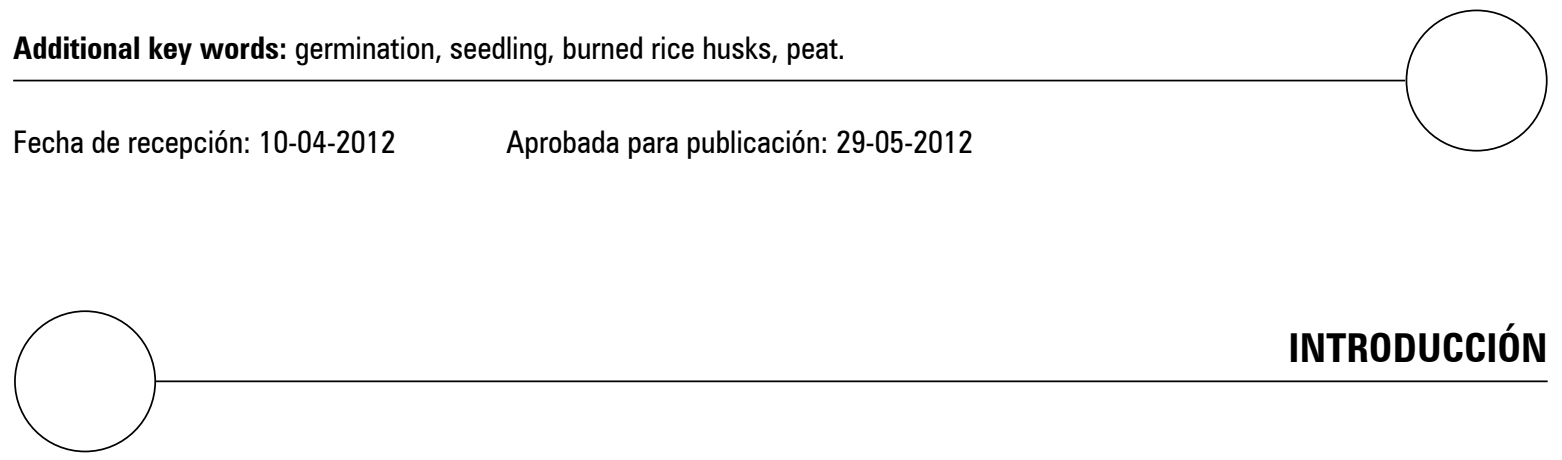

En Colombia, el área cosechada en tomate (Solanum lycopersicum L.) para el año 2010 fue de 6.508 ha, con una producción de 197.374 ha (Agronet, 2010), lo que resalta la importancia del cultivo para el país.

En el proceso de producción de tomate la primera etapa es la obtención de plántulas, lo que requiere la elaboración de semilleros que cumplan con las condiciones requeridas, para garantizar plantas vigorosas en el trasplante, puesto que de un buen almácigo depende todo el cultivo posterior (Guzmán, 2003).

Uno de los sustratos más utilizados en la propagación de tomate es la turba debido a sus condiciones fisicoquímicas, porosidad, $\mathrm{pH}$, retención de humedad y niveles nutricionales (Escobar, 2001), no obstante, comercialmente es costosa. Lo anterior ha llevado a la realización de estudios para una búsqueda de sustratos alternativos más económicos y que a la vez garanticen plantas vigorosas.

Fernández et al. (2006) evaluaron varias alternativas de sustratos para almácigos de hortalizas, utilizando diferentes mezclas de materiales y obtuvieron que el mejor sustrato para sustituir la turba fue la mezcla de compost de cachaza de caña de azúcar y aserrín de coco molida en relación 2:1. Sin embargo, existen otros sustratos como la cascarilla de arroz, el cual es el más utilizado en cultivos hidropónicos por el bajo costo, fácil transporte, lenta degradación, alta porosidad y gran facilidad de manejo a la hora de la siembra (Aponte, 1999), además puede ser una alternativa en la propagación de tomate.

Para tratar de mejorar las propiedades fisicoquímicas de la cascarilla de arroz se ha recurrido desde hace algunos años a la quema parcial de la misma. Esta es la alternativa más usada en la 
actualidad ya que aumenta la capacidad de retención de agua (Calderón et al., 2001). No obstante, uno de los mayores limitantes es el bajo contenido nutricional, lo que hace necesario complementar el sustrato para mejorar las propiedades químicas del mismo. Álvarez-Herrera et al. (2007) encontraron que la cascarilla de arroz quemada (CAO) es un sustrato que se puede utilizar en la propagación de esquejes de romero, del mismo modo, Álvarez-Herrera et al. (2008) determinaron que el uso de $\mathrm{CAQ}$ en propagación es más económico que el uso de turba rubia.

La nutrición adecuada es uno de los factores más importantes en la producción de plántulas, donde el $\mathrm{N}$ y el $\mathrm{K}$ son los nutrimentos requeridos en mayor cantidad (Marschner, 1995). Preciado et al. (2002) demostraron que soluciones nutritivas a base de nitrógeno y potasio en plántulas de dos híbridos de melón Cucumis melo L. permitieron que las plántulas presentaran mayor crecimiento y acumulación de nutrientes al momento del trasplante.

Por otro lado, se busca complementar el proceso de producción de plántulas, con la utilización de semillas que garanticen una germinación rápida, uniformidad y plantas vigorosas por lo que se muestra como alternativa la utilización de productos hormonales (Weaver, 1996). Dentro de las fitohormonas recomendadas se encuentran las giberelinas que tienen propiedades estimuladoras de la germinación, la elongación celular y la emergencia de la radícula a través del endospermo (Taylorson y Hendricks, 1977; Salisbury y Ross, 2000). Según Balaguera-López et al. (2009), al imbibir semillas de tomate en 900 $\mathrm{mg} \mathrm{L}{ }^{-1}$ de ácido giberélico $\left(\mathrm{GA}_{3}\right)$, las plántulas de tomate fueron más vigorosas, con mayor altura y diámetro de tallo.

Por lo anterior, el objetivo de esta investigación fue evaluar el efecto de la aplicación de $\mathrm{GA}_{3}$ en la germinación de semillas de tomate bajo diferentes concentraciones de fertilizantes en un sustrato de CAQ y turba, con el fin de brindar al productor una alternativa más económica en la etapa de propagación.

\section{MATERIALES Y MÉTODOS}

El experimento se realizó en el invernadero de vidrio de la Facultad de Ciencias Agropecuarias, de la Universidad Pedagógica y Tecnológica de Colombia (UPTC), sede Tunja, el cual se encuentra a una altitud de $2.782 \mathrm{msnm}$ y tiene las coordenadas $5^{\circ} 32^{\prime} \mathrm{N}$ y $73^{\circ} 23^{\prime} \mathrm{W}$; dentro del invernadero la temperatura promedio fue de $15,8^{\circ} \mathrm{C}$ y la humedad relativa promedio (HR) del $71,8 \%$. Los análisis de laboratorio se realizaron en el laboratorio de Fisiología Vegetal de la UPTC.

Para la realización del ensayo se emplearon semillas de la especie Solanum lycopersicum híbrido Calima, las cuales se imbibieron en agua con diferentes concentraciones de ácido giberélico previo a la siembra y se propagaron en bandejas de polietileno Herkupak de 144 celdas.

Como sustrato se utilizó, la mezcla de cascarilla de arroz quemada (CAO) con diferentes dosis de fertilizantes ( $\mathrm{N}, \mathrm{P}$ y $\mathrm{K}$ ) los cuales se incorporaron al sustrato en tres aplicaciones, 12, 16 y 20 días después de la siembra (dds), durante todo el proceso de germinación se utilizó turba canadiense como tratamiento testigo.

Se elaboró un diseño estadístico completamente al azar con arreglo factorial de $5 \times 5$, en donde el primer factor correspondió a las concentraciones de ácido giberélico (0, 200, 400, 600 y 800 $\mathrm{mg} \mathrm{L}^{-1}$ ) y el segundo factor correspondió a los diferentes sustratos: cascarilla quemada más la adición de distintas dosis de fertilizantes de $\mathrm{N}$, $\mathrm{P}$ y K en dosis de 90-75-180, 75-60-165, 60-45150 y 45-30-135 $\mathrm{mg} \mathrm{L}^{-1}$ de NPK y el sustrato turba como tratamiento testigo para un total de 25 tratamientos, con cuatro repeticiones, corre- 
spondiente a 100 unidades experimentales (UE) (tabla 1).

Se imbibió las semillas en agua con las diferentes concentraciones de $\mathrm{GA}_{3}$ durante $24 \mathrm{~h}$, luego se procedió a la siembra, una semilla por alveolo. La fertilización se aplicó en el agua de riego y se hizo de manera homogénea para todos los tratamientos. El programa de fertirriego incluyó aplicaciones a los 12,16 y 20 dds.

Las variables de respuesta que se determinaron fueron porcentaje de germinación (PG), tiempo medio de germinación (TMG) y velocidad media de germinación (VMG), para las cuales se hicieron observaciones diarias de las semillas hasta que emergió el primer cotiledón y se tuvo en cuenta el número de días que empleó cada semilla para germinar en un periodo de 30 dds (tabla 2). En la etapa de plántula se evaluó el área foliar mediante el analizador Li-Cor ${ }^{\circledR}$ 3000A (Li-Cor, Lincoln, NE), altura final, masa fresca de tallo, raíz y hojas, masa seca total, de tallo, raíz y hojas después de someter las plantas a $70^{\circ} \mathrm{C}$ durante $48 \mathrm{~h}$; diámetro del tallo mediante el calibrador de Vernier y la eficiencia económica de los trata-

Tabla 1. Convenciones para los diferentes tratamientos evaluados en la propagación de semillas de tomate.

\begin{tabular}{|c|c|c|c|c|c|}
\hline \multirow{2}{*}{ Tratamientos } & \multirow{2}{*}{ Sustrato } & \multicolumn{3}{|c|}{ Dosis de fertilización $\left(\mathrm{mg} \mathrm{L}^{-1}\right)$} & \multirow{2}{*}{$\begin{array}{c}\text { Dosis de GA } \\
\left(\mathrm{mg} \mathrm{L}^{-1}\right)\end{array}$} \\
\hline & & Nitrógeno & Fósforo & Potasio & \\
\hline $\mathrm{T} 1$ & CAO & 90 & 75 & 180 & 200 \\
\hline $\mathrm{T} 2$ & $\mathrm{CAO}$ & 75 & 60 & 165 & 200 \\
\hline T3 & CAO & 60 & 45 & 150 & 200 \\
\hline T4 & CAO & 45 & 30 & 135 & 200 \\
\hline $\mathrm{T} 5$ & Turba & - & - & - & 200 \\
\hline T6 & CAO & 90 & 75 & 180 & 400 \\
\hline $\mathrm{T7}$ & $\mathrm{CAO}$ & 75 & 60 & 165 & 400 \\
\hline T8 & $\mathrm{CAO}$ & 60 & 45 & 150 & 400 \\
\hline T9 & $\mathrm{CAO}$ & 45 & 30 & 135 & 400 \\
\hline $\mathrm{T} 10$ & Turba & - & - & - & 400 \\
\hline T11 & CAO & 90 & 75 & 180 & 600 \\
\hline $\mathrm{T} 12$ & $\mathrm{CAO}$ & 75 & 60 & 165 & 600 \\
\hline T13 & CAO & 60 & 45 & 150 & 600 \\
\hline $\mathrm{T} 14$ & CAO & 45 & 30 & 135 & 600 \\
\hline $\mathrm{T} 15$ & Turba & - & - & - & 600 \\
\hline $\mathrm{T} 16$ & CAO & 90 & 75 & 180 & 800 \\
\hline $\mathrm{T} 17$ & $\mathrm{CAO}$ & 75 & 60 & 165 & 800 \\
\hline T18 & CAO & 60 & 45 & 150 & 800 \\
\hline T19 & CAO & 45 & 30 & 135 & 800 \\
\hline $\mathrm{T} 20$ & Turba & - & - & - & 800 \\
\hline T21 & CAO & 90 & 75 & 180 & 0 \\
\hline $\mathrm{T} 22$ & CAO & 75 & 60 & 165 & 0 \\
\hline $\mathrm{T} 23$ & CAO & 60 & 45 & 150 & 0 \\
\hline $\mathrm{T} 24$ & CAO & 45 & 30 & 135 & 0 \\
\hline $\mathrm{T} 25$ & Turba & & & & 0 \\
\hline
\end{tabular}

$\mathrm{CAO}$, cascarilla de arroz quemada. 
Tabla 2. Fórmulas empleadas para el cálculo de las variables de germinación.

\begin{tabular}{|l|c|c|}
\hline \multicolumn{1}{|c|}{ Variable } & Ecuación & Unidades \\
\hline Velocidad media de germinación & VME $=\Sigma\left(\frac{n_{i}}{t_{i}}\right)$ & Semillas germinadas /día \\
\hline Tiempo medio de germinación & TMG $=\frac{N^{*}\left(A_{1}+A_{2}+A_{x}\right)}{\left(A_{1}{ }^{*} T_{1}+A_{2}{ }^{*} T_{2}+A_{x}{ }^{*} T_{x}\right)}$ & días \\
\hline Porcentaje de germinación & $P G=\left(\frac{N}{N_{s}}\right){ }^{*} 100$ & $\%$ \\
\hline
\end{tabular}

$\mathrm{n}_{\mathrm{i}}=$ número de semillas germinadas en el i-ésimo día;

$\mathrm{t}_{\mathrm{i}}=$ tiempo en días, para la germinación en el i-ésimo día.

$\mathrm{N}=$ número de semillas germinadas.

$A_{1}, A_{2}, \ldots, A_{x}$ : número de semillas germinadas en el día 1 , en el día $2, y$ en el día $x$;

$T_{1}, T_{2}, \ldots, T_{x}:$ número de días entre la siembra y el día 1 de germinación, entre el día 2 y entre el día $x$.

mientos: biomasa seca resultante dividido por el costo de cada tratamiento.

Los resultados de la presente investigación fueron sometidos a un análisis de varianza (Anova) con el fin de determinar diferencias significativas entre tratamientos y a pruebas de comparación de Tukey al $5 \%$ para clasificar los mismos, el software utilizado fue el SAS v. 8e.

\section{RESULTADOS Y DISCUSIÓN}

\section{Porcentaje de germinación (PG)}

De acuerdo con el análisis estadístico no se presentaron diferencias significativas entre tratamientos, debido a que hubo uniformidad en el PG de las semillas con la emergencia del primer cotiledón, similar a lo encontrado por Balaguera-López et al. (2009), no obstante, los tratamientos que contenían $\mathrm{GA}_{3}$ presentaron una germinación del 100\%, comparada con el testigo que fue del $91,6 \%$. Esto se atribuye al efecto que tienen las giberelinas en el proceso de germinación. Arteca (1996) afirma que las giberelinas han sido implicadas en el control y la promoción de la germinación en diferentes especies.

Araya et al. (2000) encontraron que la adición de $\mathrm{GA}_{3}$ en concentración de $2 \mathrm{mg} \mathrm{L}^{-1}$ en semi- llas de Jaul Alnus Acuminata bajo condiciones de luz aumentaba el PG, sin embargo, Hernández (1990) encontró que 100 y 500 mg L-1 de $\mathrm{GA}_{3}$ inhibieron la germinación de las mismas plantas en $10 \%$ y $35 \%$ respectivamente frente a $5 \mathrm{mg} \mathrm{L}^{-1} \mathrm{de}$ $\mathrm{GA}_{3}$, aunque superaron al testigo sin aplicación.

\section{Tiempo medio de germinación (TMG)}

Los resultados de esta variable mostraron que el menor TMG ocurrió a los $8 \mathrm{~d}$ en los tratamientos de $400 \mathrm{mg} \mathrm{L}^{-1}$ de $\mathrm{GA}_{3}+\mathrm{CAQ}+45-30-135$ ppm y $400 \mathrm{mg} \mathrm{L}^{-1} \mathrm{de} \mathrm{GA}_{3}+$ turba, los cuales fueron estadísticamente iguales, presentando diferencias significativas frente a los demás tratamientos donde la concentración de $600 \mathrm{mg} \mathrm{L}^{-1}$ de $\mathrm{GA}_{3}+$ $\mathrm{CAO}+90-75-180$ ppm de $\mathrm{N}$, P y K y $0 \mathrm{GA}_{3}$ en CAO+ 45-30-135 ppm de N, P y K tardaron más días (11,2 d) en germinar. Estos resultados son acordes a lo encontrado por Balaguera-López et al. (2009), quienes hallaron un TMG de 10,1 d cuando sometieron semillas de tomate a $300 \mathrm{mg}$ $\mathrm{L}^{-1}$ de $\mathrm{GA}_{3}$.

Los tratamientos a base de cascarilla presentaron mayor retraso en cuanto al tiempo de germinación con respecto a la turba (figura 1a), lo que podría asociarse con la textura y mayor porosidad de la turba, además la cascarilla de arroz quemada pudo dificultar el contacto de la semi- 
lla con este sustrato, disminuyendo la disponibilidad de agua para la germinación, lo que sugiere el manejo de láminas de riego más altas.

Handreck y Black (2002) afirman que la alta porosidad del sustrato favorece el proceso de germinación puesto que provee un buen contenido de oxígeno, el cual es requerido por las semillas para germinar. Cabe resaltar que en esta etapa de siembra los sustratos no recibieron dosis de fertilización, debido a que las semillas contienen reservas que mantienen el crecimiento y desarrollo hasta que esta es capaz de alimentarse por sí misma (Talón, 2000).

Por otro lado, las semillas que fueron tratadas con $400 \mathrm{mg} \mathrm{L}^{-1}$ de $\mathrm{GA}_{3}$ presentaron diferencias significativas al germinar en $9 \mathrm{~d}$ con respecto al testigo (10,8 d) (figura 1b), lo que coincide con Bewley (1997) quien afirma que las giberelinas aceleran el proceso de germinación. Además se les atribuye que junto con las auxinas influyen de forma indirecta en la absorción de agua mediante el transporte activo al aumentar la elasticidad de la pared celular (Almanza,
2000). Peñapareja et al. (2007) encontraron que la aplicación de $\mathrm{GA}_{3}$ favoreció la germinación en Peonia broteroi y disminuyó el tiempo medio de germinación, consiguiéndose el menor valor con la dosis de $250 \mathrm{mg} \mathrm{L}^{-1}$.

\section{Velocidad media de germinación (VMG)}

Los resultados mostraron que la mayor VMG fue de 0,74 semillas/día, esta ocurrió en los sustratos turba y CAO cuando se aplicó $400 \mathrm{mg} \mathrm{L}^{-1}$ de $\mathrm{GA}_{3}$ (figura 2a). Estos tratamientos presentaron diferencias significativas frente a los demás, en particular, la concentración de $800 \mathrm{mg} \mathrm{L}^{-1}$ de $\mathrm{GA}_{3}$ y CAQ mostró el menor promedio con 0,4 semillas germinadas/día (figura 2), estos datos son inferiores a los encontrados por BalagueraLópez et al. (2009) de 1,02 semillas germinadas por día con la imbibición de semillas en $300 \mathrm{mg}$ $\mathrm{L}^{-1}$ de $\mathrm{GA}_{3}$.

La concentración de $400 \mathrm{mg} \mathrm{L}^{-1}$ de $\mathrm{GA}_{3}$ con el sustrato turba indujo mayor VMG al compararse con el testigo sin aplicación de $\mathrm{GA}_{3} \mathrm{y}$ con el sustrato cascarilla de arroz quemada enriquecido

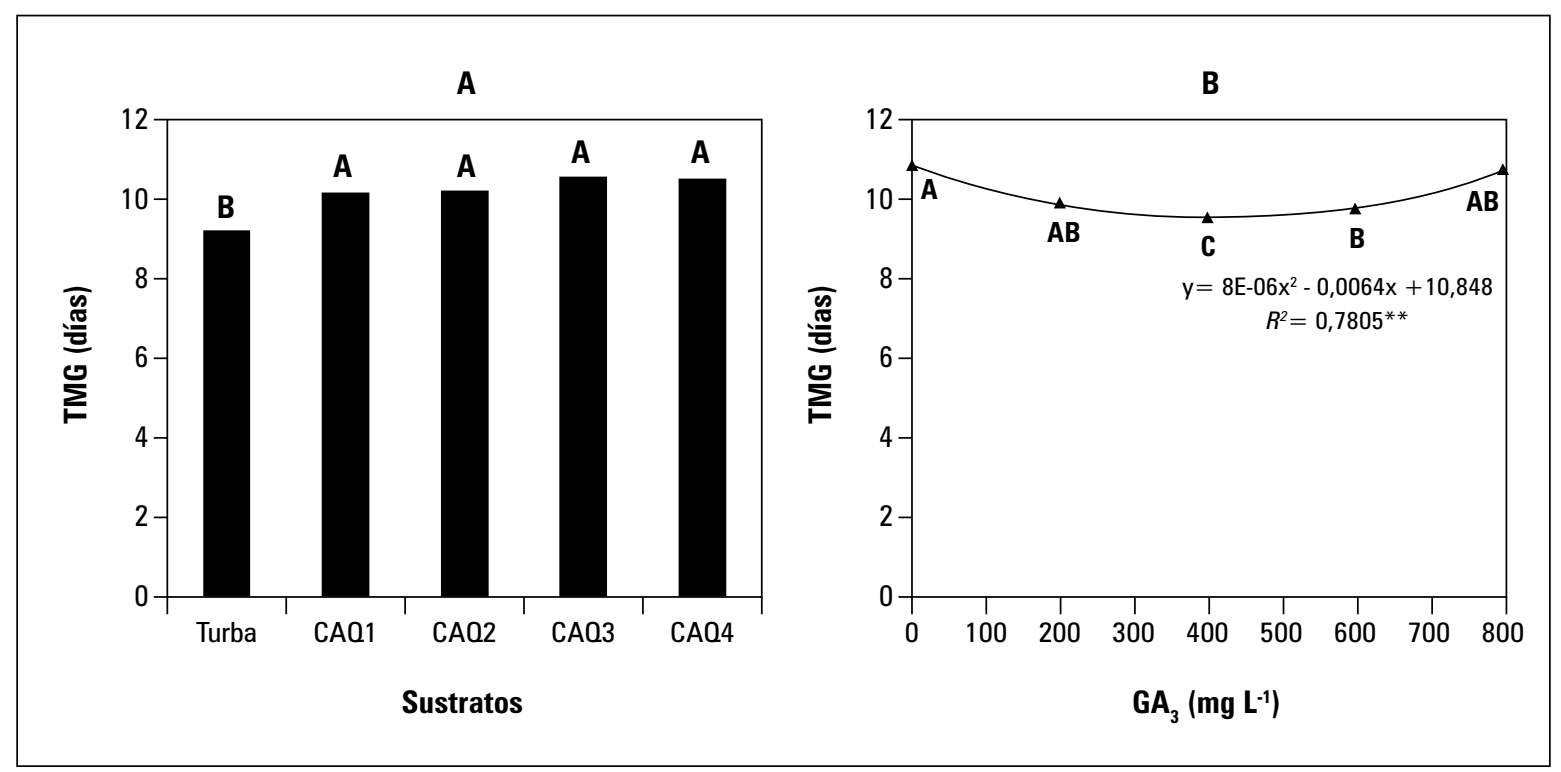

Figura 1. Tiempo medio de germinación de semillas de tomate sembradas $A$ : en diferentes sustratos CA01: CAQ + 45-30-135; CA02: CAO + 60-45-150; CA03: CAO + 75-60-165; CA04: CAO + 90-75-180; B: embebidas en diferentes concentraciones de $\mathbf{G A}_{3}$. Promedios con letras distintas indican diferencia significativa entre sustratos y concentraciones de $\mathrm{GA}_{3}$ según la prueba de Tukey $(P \leq 0,05)$. 
con 60-45-150 ppm de N, P y K (figura 2b). Esto probablemente ocurre debido a que las $\mathrm{GA}_{3}$ activan el crecimiento vegetativo del embrión, debilitando la capa del endospermo que rodea al embrión y movilizando las reservas almacenadas en el endospermo (Taiz y Zeiger, 2006). Además la aplicación de giberelinas estimula la producción de numerosas hidrolasas, sobre todo la enzima $\alpha$-amilasa la cual se mueve al endospermo donde convierte el almidón en azúcar, el cual es traslocado a los puntos de crecimiento del embrión con el fin de alimentarlo e iniciar el crecimiento de las plántulas (Davies, 2004).

\section{Altura final}

La altura final de las plántulas presentó diferencias significativas entre tratamientos. La aplicación de $400 \mathrm{mg} \mathrm{L}^{-1}$ de $\mathrm{GA}_{3}$ en el sustrato turba presentó mejor respuesta con $10 \mathrm{~cm}$ de longitud frente a los demás tratamientos, la aplicación de $800 \mathrm{mg} \mathrm{L}^{-1}$ de $\mathrm{GA}_{3}$ en el sustrato CAQ enriquecido con 90-75-180 ppm de N, P y K, tan solo presentó una altura de $2,9 \mathrm{~cm}$, siendo el tratamiento menos favorable (tabla 3 ), este resultado se puede explicar debido a que el primer tratamiento fue el que tuvo mayor VMG.

Los tratamientos con el sustrato turba presentaron un mayor incremento en la altura de las plantas, estas diferencias con la CAO se pueden atribuir a la baja retención de humedad de la CAQ, lo que pudo causar un estrés por déficit hídrico y consecuentemente una reducción del crecimiento (Sánchez-Díaz y Aguirreolea, 1996), el tratamiento sembrado en CAQ + 90-75-180 de N, P y K presentó los valores mas bajos en altura. Estos resultados coinciden con Fernández et al. (2006), quienes encontraron que en turba, las variables de crecimiento presentaron los valores más altos con respecto a otros sustratos, respuesta que estuvo muy relacionada con la dinámica de germinación presentada bajo cada uno de estos.

La dosis de fertilizante de 90-75-180 de N, P y $\mathrm{K}$ en el sustrato de CAO presentó bajos resultados en altura de planta con respecto al sustrato turba, esto pudo deberse a que la turba favorece la toma y asimilación de nutrientes a través de

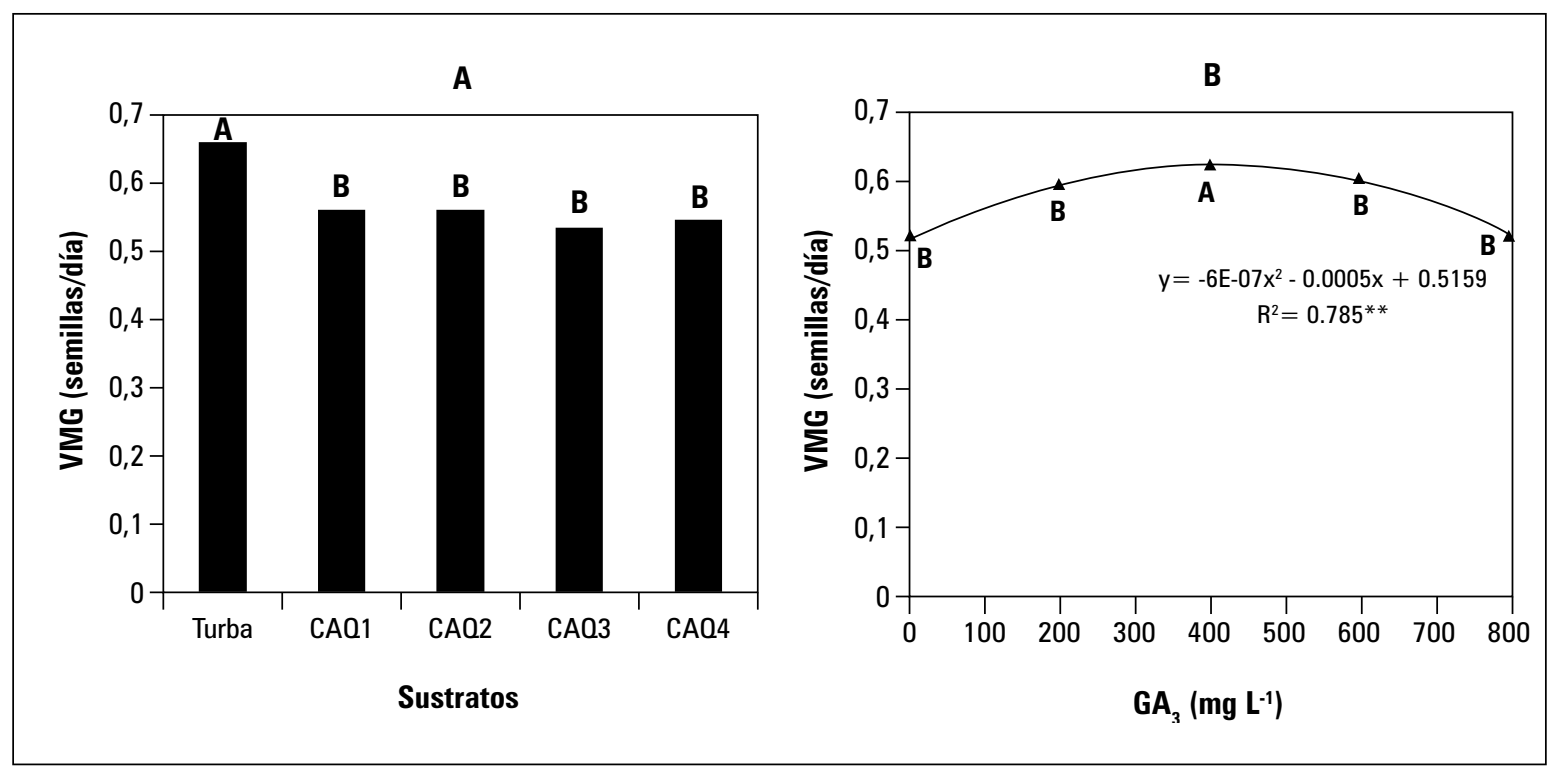

Figura 2. Velocidad media de germinación de semillas de tomate sembradas $A$ : en diferentes sustratos enriquecidos con N, P y K, así CAQ1: CAQ + 45-30-135; CA02: CAQ + 60-45-150; CAQ3: CAQ + 75-60-165; CAQ4: CAQ + 90-75-180; $B$ : embebidas en diferentes concentraciones de $\mathbf{G A}_{3}$. Promedios con letra distinta indican diferencia significativa entre sustratos y concentraciones de $\mathrm{GA}_{3}$ según la prueba de Tukey $(P \leq 0,05)$. 


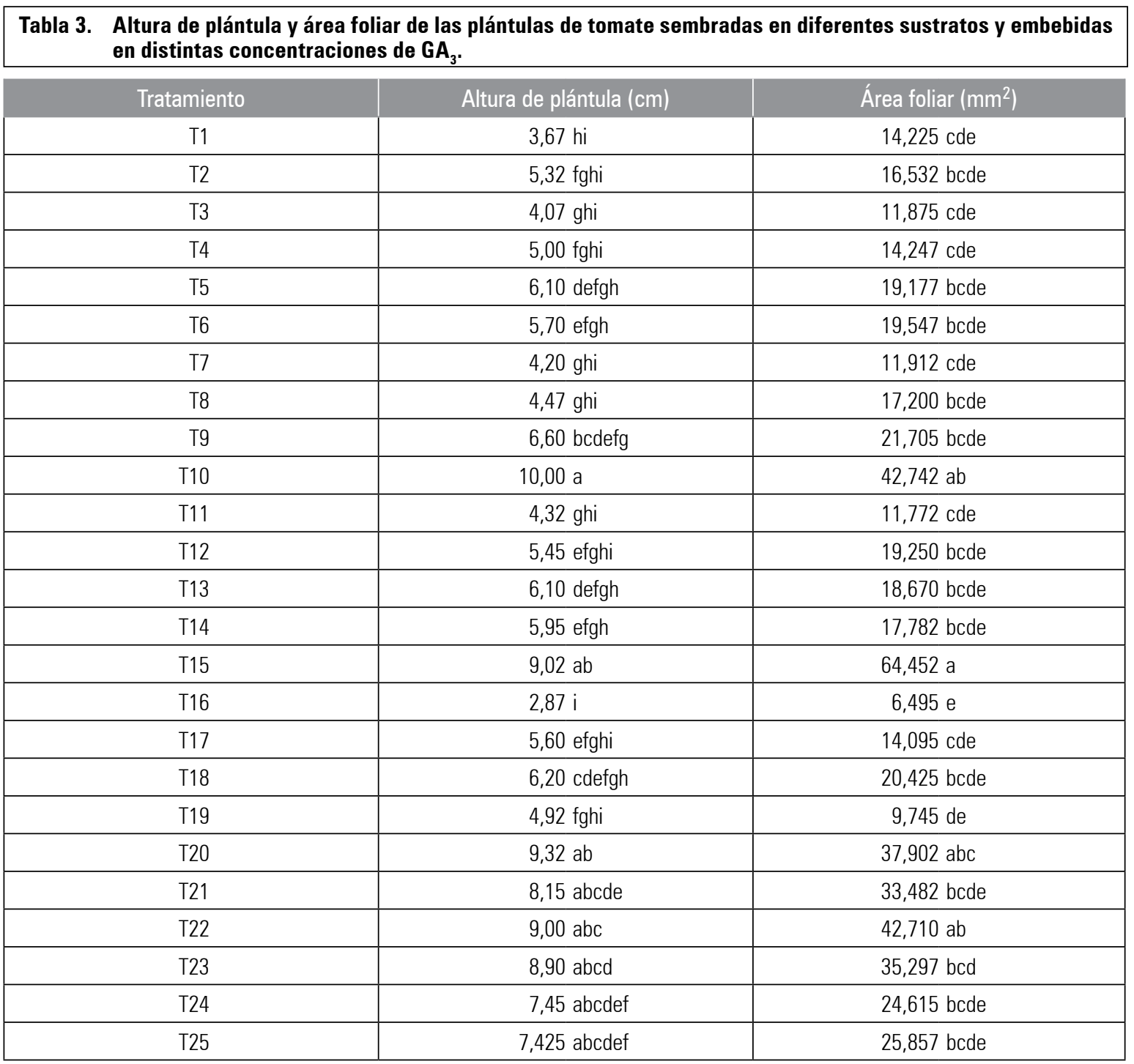

Promedios con letras distintas indican diferencia significativa entre tratamientos por columna según la prueba de Tukey $(P \leq 0,05)$.

la raíz y a la capacidad de retención de agua del sustrato ya que estas aplicaciones se hicieron utilizando fertirriego.

El crecimiento en altura de una planta depende principalmente del aporte de agua, nutrientes, energía, y aire que un sustrato pueda aportar (Scagel, 1995), ya que las condiciones fisicoquímicas de cada sustrato pueden definir el comportamiento tanto de la altura como de las demás variables agronómicas (Schnelle y Henderson, 1991).

\section{Diámetro del tallo}

Las mejores combinaciones de tratamientos con respecto al diámetro del tallo fueron las aplicaciones de concentraciones de 400 y $800 \mathrm{mg} \mathrm{L}^{-1}$ de $\mathrm{GA}_{3}$ sembradas en turba, estas fueron estadísticamente iguales con $3,1 \mathrm{~mm}$, frente a 600 y 800 $\mathrm{mg} \mathrm{L}^{-1}$ de $\mathrm{GA}_{3}$ sembradas en $\mathrm{CAQ}+90-75-180$ $\mathrm{N}, \mathrm{P}$ y K con 1,5 mm (figura 3).

Aunque las concentraciones de $\mathrm{GA}_{3}$ no muestran una tendencia marcada en los resultados, 


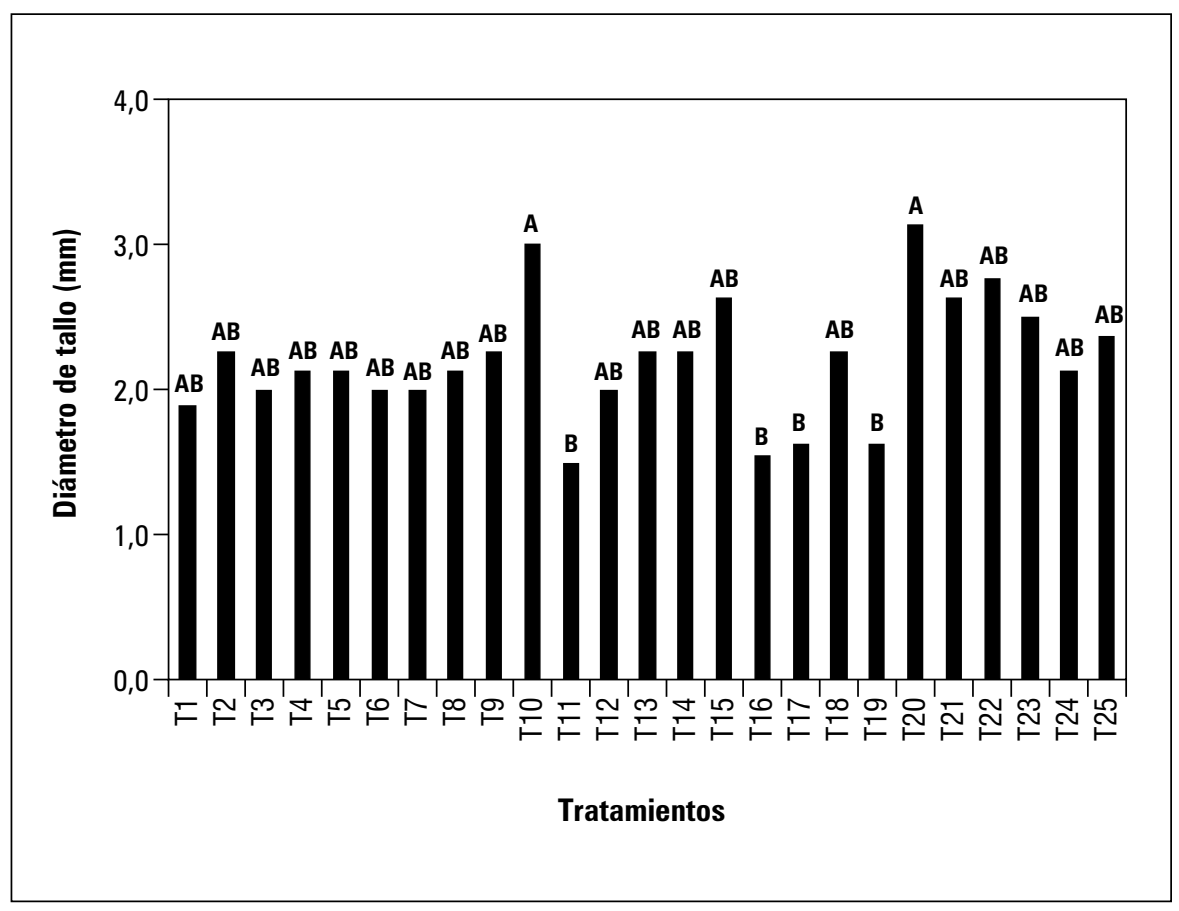

Figura 3. Diámetro de tallo de plántulas de tomate provenientes de semillas tratadas en diferentes sustratos y con diferentes dosis, como indica la tabla 1.

se puede apreciar que para este caso es el sustrato el que tiene una mayor influencia ya que la turba presentó mejores resultados en general, a pesar del contenido nutricional que se le adicionó a la cascarilla de arroz quemada.

La dosis más alta de fertilización (90-75-180) presentó los resultados menos favorables en grosor de tallo, lo que se puede atribuir a estrés por toxicidad lo cual redujo el crecimiento de la plántula y con ello su metabolismo. No obstante, Basocuu y Nicola (1995) encontraron que las fertilizaciones con nitrógeno afectan favorablemente el diámetro del tallo y el número de hojas de las plántulas, sin embargo en el presente estudio el factor que más efecto presentó fue el sustrato.

El grosor de tallo es un indicador del estado de vigor de una plántula (Quesada et al., 2005) ya que refleja directamente la acumulación de fotosintetizados, los cuales posteriormente pueden traslocarse a los vertederos (Donald y Hamblin, 1983), además un tallo grueso permite soportar la parte aérea sin doblarse por los vientos en el campo (Orzolek, 1991).

\section{Longitud de raíz principal}

La respuesta en crecimiento de la longitud de raíz principal no se vio afectada significativamente por el sustrato ni por la concentración de $\mathrm{GA}_{3}$, sin embargo la concentración de $600 \mathrm{mg} \mathrm{L}^{-1}$ de $\mathrm{GA}_{3}$ y 60-45-150 de N, P y K presentó el mayor promedio con $13,22 \mathrm{~cm}$, este resultado es similar al obtenido por Wilches-Rojas et al. (2008), quienes obtuvieron en plántulas de tomate una longitud de raíz principal de 11,97 cm al evaluar láminas de riego y tamaños de alveolo en diferentes híbridos de tomate.

Ningún sustrato ni concentración de $\mathrm{GA}_{3}$ presentó una influencia significativa sobre la longitud de la raíz, resultado que se puede atribuir a que el tiempo de la etapa de germinación es muy corto y los tratamientos no alcanzan a provocar un efecto significativo sobre estas variables, no 
obstante, se pudo apreciar un buen desarrollo radicular.

\section{Área foliar}

De acuerdo al análisis estadístico, se presentaron diferencias significativas entre tratamientos para esta variable. Los valores más altos de área foliar se obtuvieron con la concentración de 600 mg L $\mathrm{L}^{-1}$ de $\mathrm{GA}_{3}$ y turba con $64,25 \mathrm{~cm}^{2}$ frente a la concentración de $800 \mathrm{mg} \mathrm{L}^{-1}$ de $\mathrm{GA}_{3}$ y CAQ + 90-75-180 N, P y K con 6,49 $\mathrm{cm}^{2}$ (tabla 3). Este resultado se puede atribuir principalmente al sustrato CAQ, el cual presentó el menor desempeño combinado con las dosis de fertilizante, las cuales no presentaron diferencias significativas.

El mejor resultado con respecto al área foliar fue la concentración de $\mathrm{GA}_{3}$ con dosis de $600 \mathrm{mg} \mathrm{L}^{-1}$, el testigo mostró los resultados más bajos, lo cual pudo estar influenciado por el medio en el cual se sembró, ya que con la turba se obtuvieron los mejores resultados al compararlo con los resultados del sustrato CAO enriquecido con las dosis de N, P y K, por lo que se puede afirmar que el sustrato favorece el efecto de la aplicación de $\mathrm{GA}_{3}$ en el área foliar de las plántulas de tomate.

Balaguera et al. (2007) encontraron que con dosis de $300 \mathrm{mg} \mathrm{L}^{-1}$ de $\mathrm{GA}_{3}$ y 36 h de imbibición las plántulas de tomate mostraban diferencias significativas positivas con respecto al área foliar en los primeros 30 días. En Helianthus annuus L., la aplicación de $\mathrm{GA}_{3}$ al 10\% disminuyó el área foliar significativamente respecto al testigo, principalmente debido al déficit en la hidratación de las hojas, ya que las giberelinas determinan una menor tolerancia a la sequía, adicionalmente, es probable que la planta emplee una buena cantidad de nutrientes en la elongación celular del tallo, lo que disminuye el área foliar (Silva et al., 2001).

El área foliar tiene un papel muy importante en el proceso fotosintético ya que a mayor área foliar mayor captación de energía lumínica y fotoasimilados. Según Jarma et al. (1999), las plantas con mayor área foliar y ambiente favorable, son capaces de aprovechar mejor la energía solar dando como resultado una fotosíntesis más eficiente.

\section{Masa fresca total de hojas, tallo y raíz}

Según el análisis estadístico la masa fresca de hojas, tallo y raíz presentó diferencias significativas entre tratamientos, para hojas, las plántulas con mejor resultado fueron las provenientes de semillas imbibidas en $800 \mathrm{mg} \mathrm{L}^{-1}$ de $\mathrm{GA}_{3}$ y sembradas en turba, con 1,33 g, en cuanto a la masa de tallos, la imbibición en $400 \mathrm{mg} \mathrm{L}^{-1}$ de $\mathrm{GA}_{3}$ y siembra en turba presentó los valores más altos con $0,64 \mathrm{~g}$, mientras que el mayor valor de masa fresca de raíces se presentó cuando las semillas fueron imbibidas en agua y sembradas en $\mathrm{CAQ}+75-60-165$ de $\mathrm{N}$, P y K (tabla 4).

Pese a que no se observa ninguna tendencia marcada en estas tres variables se ve que los tratamientos de $400 \mathrm{mg} \mathrm{L}^{-1}$ de $\mathrm{GA}_{3}+$ turba y 800 $\mathrm{mg} \mathrm{L^{-1 }}+$ turba, presentan los mejores resultados frente a los demás; ya que en toda la etapa mostraron un mayor vigor de acuerdo con las variables evaluadas, lo que pudo aumentar así la capacidad de acumulación de osmolitos, que garantizan un mejor crecimiento y, por tanto, mayor producción de fotoasimilados y de masa fresca (Salisbury y Ross, 1994).

El tratamiento con $800 \mathrm{mg} \mathrm{L}^{-1}+\mathrm{CAQ}$, enriquecido con 90-75-180 de N, P y K, fue el que presentó menor desempeño en hojas, tallo y raíz mostrando así la misma tendencia en la altura final, área foliar y número de foliolos. Este resultado se puede asociar al contenido de humedad del sustrato, ya que la masa fresca depende de la turgencia de la planta y de la humedad del medio en el momento de la toma de la muestra. García et al. (2001) encontraron rendimien- 


\begin{tabular}{|c|c|c|c|c|c|c|}
\hline Tratamiento & $\begin{array}{c}\text { Masa fresca } \\
\text { hojas }(\mathrm{g})\end{array}$ & $\begin{array}{c}\text { Masa fresca } \\
\text { tallos }(\mathrm{g})\end{array}$ & $\begin{array}{c}\text { Masa fresca } \\
\text { raíz }(\mathrm{g})\end{array}$ & $\begin{array}{l}\text { Masa seca } \\
\text { hojas }(\mathrm{g})\end{array}$ & $\begin{array}{l}\text { Masa seca } \\
\text { tallos }(\mathrm{g})\end{array}$ & $\begin{array}{c}\text { Masa seca raíz } \\
(\mathrm{g})\end{array}$ \\
\hline $\mathrm{T} 1$ & 0,293 ef & 0,135 ef & $0,153 \mathrm{bc}$ & 0,026 bc & $0,007 \mathrm{~b}$ & 0,026 a \\
\hline $\mathrm{T} 2$ & 0,328 def & 0,165 def & $0,185 \mathrm{bc}$ & $0,027 \mathrm{bc}$ & $0,030 \mathrm{ab}$ & $0,010 \mathrm{a}$ \\
\hline T3 & 0,243 ef & $0,093 \mathrm{f}$ & $0,123 \mathrm{bc}$ & 0,039 bc & $0,012 \mathrm{~b}$ & $0,015 \mathrm{a}$ \\
\hline T4 & 0,400 def & 0,165 def & $0,200 a b c$ & $0,015 \mathrm{c}$ & $0,006 \mathrm{~b}$ & $0,007 \mathrm{a}$ \\
\hline T5 & 0,483 cdef & 0,208 cdef & $0,280 a b c$ & $0,063 a b c$ & $0,014 a b$ & $0,021 \mathrm{a}$ \\
\hline T6 & 0,520 cdef & 0,198 cdef & $0,345 a b c$ & $0,053 \mathrm{bc}$ & $0,015 a b$ & $0,025 \mathrm{a}$ \\
\hline $\mathrm{T} 7$ & 0,270 ef & 0,110 ef & $0,178 \mathrm{bc}$ & $0,022 \mathrm{bc}$ & $0,005 \mathrm{~b}$ & $0,009 a$ \\
\hline T8 & 0,410 def & 0,143 ef & $0,305 a b c$ & $0,039 \mathrm{bc}$ & $0,009 \mathrm{~b}$ & $0,018 \mathrm{a}$ \\
\hline T9 & 0,563 bcdef & 0,263 bcdef & $0,355 a b c$ & 0,056 bc & $0,015 a b$ & $0,028 \mathrm{a}$ \\
\hline $\mathrm{T} 10$ & $1,255 a b$ & $0,645 \mathrm{a}$ & $0,410 a b c$ & $0,125 a b$ & $0,037 \mathrm{ab}$ & $0,032 \mathrm{a}$ \\
\hline T11 & 0,243 ef & 0,098 ef & $0,143 \mathrm{bc}$ & $0,021 \mathrm{bc}$ & $0,007 \mathrm{~b}$ & $0,007 \mathrm{a}$ \\
\hline $\mathrm{T} 12$ & 0,490 cdef & 0,170 def & $0,338 \mathrm{abc}$ & $0,046 \mathrm{bc}$ & $0,011 \mathrm{~b}$ & $0,021 \mathrm{a}$ \\
\hline $\mathrm{T} 13$ & 0,475 cdef & 0,190 cdef & $0,338 a b c$ & $0,043 \mathrm{bc}$ & $0,013 \mathrm{ab}$ & 0,021 a \\
\hline T14 & 0,480 & 0,205 cdef & $0,360 a b c$ & $0,043 \mathrm{bc}$ & $0,012 b$ & $0,020 \mathrm{a}$ \\
\hline T15 & 0,948 cdef & $0,445 \mathrm{abcd}$ & $0,463 a b c$ & $0,168 \mathrm{a}$ & $0,046 \mathrm{a}$ & $0,044 \mathrm{a}$ \\
\hline T16 & 0,100 abcde & $0,048 \mathrm{f}$ & $0,048 \mathrm{c}$ & $0,051 \mathrm{bc}$ & $0,006 \mathrm{~b}$ & $0,011 \mathrm{a}$ \\
\hline $\mathrm{T} 17$ & 0,283 ef & 0,143 ef & $0,178 \mathrm{bc}$ & $0,027 \mathrm{bc}$ & $0,008 \mathrm{~b}$ & $0,015 \mathrm{a}$ \\
\hline $\mathrm{T} 18$ & 0,473 cdef & 0,203 cdef & $0,243 a b c$ & $0,044 \mathrm{bc}$ & $0,012 b$ & $0,017 \mathrm{a}$ \\
\hline T19 & 0,228 ef & 0,120 ef & $0,203 a b c$ & $0,020 \mathrm{bc}$ & $0,007 \mathrm{~b}$ & $0,011 \mathrm{a}$ \\
\hline T20 & $1,330 \mathrm{a}$ & $0,523 a b$ & $0,568 \mathrm{ab}$ & $0,124 a b$ & $0,031 \mathrm{ab}$ & $0,038 \mathrm{a}$ \\
\hline T21 & 0,913 abcde & 0,390 abcde & $0,528 a b c$ & $0,099 a b c$ & $0,029 a b$ & $0,040 \mathrm{a}$ \\
\hline $\mathrm{T} 22$ & $1,183 \mathrm{abc}$ & $0,458 \mathrm{abcd}$ & 0,688 a & $0,084 \mathrm{abc}$ & $0,030 \mathrm{ab}$ & $0,048 \mathrm{a}$ \\
\hline $\mathrm{T} 23$ & $1,033 \mathrm{abcd}$ & $0,478 a b c$ & $0,500 \mathrm{abc}$ & $0,096 a b c$ & $0,026 a b$ & $0,039 a$ \\
\hline T24 & 0,678 abcdef & 0,313 bcdef & $0,293 a b c$ & $0,060 a b c$ & $0,016 a b$ & $0,019 a$ \\
\hline T25 & 0,638 abcdef & 0,263 bcdef & $0,363 a b c$ & $0,079 a b c$ & $0,018 a b$ & 0,024 a \\
\hline
\end{tabular}

Promedios con letras distintas indican diferencia significativa entre tratamientos por columna según la prueba de Tukey $(P \leq 0,05)$. Abreviaturas de los tratamientos ver tabla 1.

tos bajos en plantas de Epipremnum aureum L. sembradas en sustratos a partir de cascarilla de arroz lo que atribuyeron a la capacidad de retención de humedad.

Pizano de Márquez (2000) y Ramírez (2000) afirman que la cascarilla es un sustrato reactivo por su $\mathrm{pH}$, debido a los óxidos derivados del proceso de quemado, lo que afecta la disponibilidad de la mayoría de los elementos. Sin embargo, este sustrato es muy utilizado en hidroponía y presenta buenos resultados por su alta porosidad (Castrillón, 2000; De Boodt et al., 1974).

\section{Masa seca total de tallo, raíz y hojas}

Estadísticamente se encontraron diferencias entre tratamientos. La imbibición de las semillas en $600 \mathrm{mg} \mathrm{L}^{-1}$ de $\mathrm{GA}_{3}$ y su posterior siembra en turba, originaron en las plántulas de tomate la mayor acumulación de masa seca tanto en hojas como en tallos, con valores de 0,16 y 0,045 g res- 
pectivamente, este tratamiento fue seguido por los tratamientos sembrados en turba con aplicación de 400 y $800 \mathrm{mg} \mathrm{L}^{-1}$ de $\mathrm{GA}_{3}$ (tabla 4), los cuales presentaron a su vez, la mayor acumulación de masa seca de raíz. Las concentraciones de $\mathrm{GA}_{3}$ presentaron diferencias significativas, los valores más altos de masa seca para todos los órganos se obtuvieron con la imbibición de las semillas de tomate en las dosis de 400, $600 \mathrm{y}$ $800 \mathrm{mg} \mathrm{L}^{-1}$ de $\mathrm{GA}_{3}$.

El sustrato de CAQ con la dosis de 45-30-135 mg L-1 de N, P y K tuvo los rendimientos más bajos con respecto a la turba, debido posiblemente a la baja concentración de nutrientes que le aportó el sustrato a la planta, además si existe una limitada disponibilidad de agua esta influye en la movilidad de nutrientes, por lo que un contenido bajo de nutrientes puede limitar el crecimiento de la raíz y por consiguiente afectar el tallo, ya que los nutrientes son inicialmente absorbidos por los pelos radiculares y luego se traslocan al tallo y a los demás órganos (Epstein y Bloom, 2005).

Según Páez et al. (2000), la cantidad de masa seca producida por una planta individual es un indicador de su capacidad de utilización de los recursos disponibles para el crecimiento vegetal. El mayor contenido de masa seca se almacenó en las hojas indicando que para esta época, estas demandan mayor cantidad de fotoasimilados a diferencia del tallo y la raíz, de igual forma se evidenció una relación positiva directamente proporcional con respecto al área foliar ya que un aumento en la masa seca de tallo afectó de manera positiva la masa seca de raíz.

\section{CONCLUSIONES}

La imbibición de las semillas de tomate en 400 $\mathrm{mg} \mathrm{L}^{-1}$ de $\mathrm{GA}_{3}$ indujo un menor tiempo medio de germinación y mayor velocidad media de germinación. El porcentaje de germinación fue del $100 \%$ en los tratamientos con $\mathrm{GA}_{3}$ y en el sustrato de turba, con respecto al 91,6\% del testigo en el sustrato de cascarilla de arroz quemada y las dosis de N, Py K.

Las plantas sembradas en el sustrato de turba con una concentración de $400 \mathrm{mg} \mathrm{L}^{-1} \mathrm{GA}_{3}$ mostraron un mayor vigor lo cual se expresó en la altura, diámetro de tallo, fitomasa fresca y fitomasa seca.

A pesar de enriquecer el sustrato CAQ con fertilización, las plántulas sembradas en este presentaron un desarrollo inferior con respecto a las sembradas en turba.

Las evaluaciones de las variables mostraron un mayor efecto del sustrato que de la imbibición de las semillas de tomate en ácido giberélico en el vigor y el desarrollo de las plántulas de tomate.

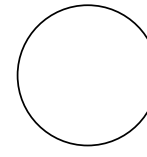

Agronet. 2010. Análisis estadístico de área y producción agrícola y pecuaria. En http://www. agronet.gov.co/agronetweb1/Estadísticas/ReportesEstadísticos.aspx; consulta: noviembre de 2011.

Almanza, P. 2000. Fisiología vegetal. Instituto Universitario Juan de Castellanos, Tunja, Colombia.

\section{REFERENCIAS BIBLIOGRÁFICAS}

Álvarez-Herrera, J.G., S.L. Rodríguez y E. Chacón. 2007. Efecto de diferentes tamaños de esqueje y sustratos en la propagación del romero (Rosmarinus officinalis L.). Agron. Colomb. 25(2), 224-230.

Álvarez-Herrera, J.G., E. Chacón y S.L. Rodríguez. 2008. Efecto de dos sustratos y diferentes láminas de riego en la propagación de romero (Rosmarinus offi- 
cinalis L.). Rev. UDCA Actual. Divulg. Cient. 11(1), 103-111.

Aponte, A. 1999. Cultivos protegidos con técnica hidropónica y biológica, Tomo I: Cómo producir 350 ton/ha/cosecha de tomate en cualquier clima. Bogota.

Araya, E., L. Gómez, N. Hidalgo y R. Valverde. 2000. Efecto de la luz y del ácido giberélico sobre la germinación in vitro de jaul (Alnus acuminata). Agron. Costarricense 24(1), 75-80.

Arteca, R. 1996. Plant growth substances. Principles and aplications. Chapman and Hall, New York, NY.

Balaguera, E., D. Rodríguez y J. Álvarez. 2007. Aplicación de giberelinas $\mathrm{GA}_{3} \mathrm{y}$ diferentes tiempos de imbibición en semillas de tomate (Lycopersicon esculentum Mill). p.178. En: Memorias II Congreso Colombiano de Horticultura. Editorial Produmedios, Bogotá.

Balaguera-López, H.E., J.F. Cárdenas-Hernández y J.G. Álvarez-Herrera. 2009. Effect of gibberellic acid $\left(\mathrm{GA}_{3}\right)$ on seed germination and growth of tomato (Solanum lycopersicum L.). Acta Hort. 821, 141-147.

Basocuu, L. y S. Nicola. 1995. Suplementtary light and pretransplant nitrogen effects on tomato seedling growth and yield. Acta Hort. 396, 313-396

Bewley, J. D. 1997. Seed germination and dormancy. Plant Cell 9, 1055-1066.

Burgas, R. y A. Powell. 1984. Evidence for repair processes in the invigoration of seeds by hydration. Ann. Bot. 53, 753-757.

Calderón, F. y F. Cevallos. 2001. Los sustratos. En: www. drcalderonlabs.com. Bogotá D.C., Colombia; consulta: noviembre de 2011.

Castrillón, I. 2000. Última tecnología en sustratos para enraizamiento. Acopaflor 7(2), 35.

Davies, P. 2004. Plants hormones. Kluwer Academic Publishers, New York, NY.

De Boodt, M., O. Verdonck y I. Cappaert. 1974. Method for measuring the water release curve of organic substrates. Acta Hort. 37, 2054-2062.

Donald, C.M. y J. Hamblin. 1983. The convergent evolution of annual seed crops in agriculture. Adv. Agron. 36, 97-143.

Epstein, E. y A.J. Bloom. 2005. Mineral nutrition of plants: principles and perspectives. Sinauer Associates Publishers, Sunderland, MA.

Escobar, H. 2001. Generalidades del cultivo. pp. 13-19. En: Escobar, H. y R. Lee (eds.). Producción de to- mate bajo invernadero. Fundación Universidad de Bogotá, Jorge Tadeo Lozano, Bogotá.

Fernández, B.C., N. Urdanet y W. Silva. 2006. Germinación de semillas de tomate (Lycopersicum esculentum Mill.) cv. Río Grande sembradas en bandejas plásticas, utilizando distintos sustratos. Rev. Fac. Agron. 23(2), 188-196.

García, O., G. Alcantar, R.I. Cabrera, R. Gavi y H. Volke. 2001. Evaluación de sustratos para la producción de Epipremnum aureum y Spathiphyllum wallisii cultivadas en maceta. Terra 19, 249-258.

Guzmán, J. M. 2003. Sustratos y tecnología de almácigo. p. 25. En: Memoria de cursos de producción en ambientes protegidos. UCR-CYTED, San José, Costa Rica.

Handreck, K.A. y N. Black. 2002. Growing media for ornamental plants and turf. 3 ed. UNSW Press, Sydney.

Hernández, R. 1990. Un estudio sobre la germinación de la semilla de Alnus acuminata H.B.K. Rev. Forestal Venezolana 3, 39-54.

Hartmann, H.T. y D.E. Kester. 1989. Propagación de plantas. Compañía Editorial Continental S.A., México.

Jarma, A., C. Buitrago y S. Gutiérrez. 1999. Respuesta del crecimiento de la habichuela (Phaseolus vulgaris L. var. Blue Lake) a tres niveles de radiación incidente. Revista Comalfi 26(1-3), 62-73.

Marschner, H. 1995. Mineral nutrition of higher plants. Academic Press, San Diego, CA.

Observatorio de finanzas rurales. Tomate. 2012. Disponible en: http://www.agronet.gov.co/www/ htm3b/excepciones/cargaNet/netcarga16.aspx?co $\mathrm{d}=16 \&$ reporte $=$ Producci\%u00f $3 \mathrm{n}+$ nacional + por + producto\&file $=2007816102236 \_20058417048$ agronetevaarearendimientoyproduccionpor producto poragno.rpt\&código $=16 \&$ excepcion $=1 \& \mathrm{fe}$ chaI $=2005$ \&producto=Tomate\& Fecha $F=2005$; consulta: febrero de 2012.

Orzolek, M.D. 1991. Establishment of vegetables in the field. Hort Technol. 1, 78-81.

Páez, A., V. Paz y J. C. López. 2000. Crecimiento y respuestas fisiológicas de plantas de tomate cv. Río Grande en la época mayo-julio. Efecto del sombreado Rev. Fac. Agron. (LUZ) 17, 173-184.

Peñapareja, D., P. Sánchez-Gómez, J. López, A. González, J. Franco y J. Fernández, J. 2007. Influencia de la aplicación de ácido giberélico y el tiempo de almacenamiento en la germinación de Peonia broteroi. 
Actas de Horticultura (Sociedad Española de Ciencias Hortícolas) 48(2), 17-24.

Pizano de Márquez, M. 2000. Clavel Dianthus caryophillus. Ed. Hortitecnia Ltda., Bogotá.

Preciado, P., G. Baca, L. Tirado, J. Kohashi-Shibata, L. Tijerina y A. Martínez. 2002. Nitrógeno y potasio en la producción de plántulas de melón. Terra 20(3), 267-276.

Quesada, G. y C. Méndez. 2005. Evaluación de sustratos para almácigos de hortalizas. Agron. Mesoamer. 16(2), 171-183.

Ramírez, V.M. 2000. Caracterización de las propiedades de 10 materiales, descripción de uso potencial como sustratos y evaluación del crecimiento de plántulas de lechuga (Lactuca sativa L.) en los materiales promisorios como sustratos. Trabajo de grado. Facultad de Agronomía. Universidad Nacional de Colombia, Bogotá.

Salisbury, F.B. y C.W. Ross. 1994. Fisiología vegetal. Grupo Editorial Iberoamérica S.A., México.

Salisbury, F.B. y C.W. Ross. 2000. Fisiología de las plantas. Editorial Paraninfo Thomson Learning, Madrid.

Sánchez-Díaz, M.yJ. Aguirreolea. 1996. Relaciones hídricas. En: Azcón-Bieto, J. y M. Talón (eds.). Fisiología y bioquímica Vegetal. Edigrafos, Madrid. pp. 49-90.
Scagel, R., R.J. Bandoni, C.E. Rouse, W.B. Schofield, J.R. Stein y T.M.C. Taylor. 1995. El reino vegetal. $3^{\text {a }}$ ed. Omega, Barcelona, España.

Schnelle, M. A. y J.C. Henderson. 1991. Containers and media for the nursery. Oklahoma cooperative extension service. Extension facts. Oklahoma State University, Stillwater, OK.

Silva, M., H. Gámez, F. Zabala, B. Cuevas y M. Rojas. 2001. Efecto de cuatro fitorreguladores comerciales en el desarrollo y rendimiento del girasol. Ciencia UANL 4(1), 69-75.

Taiz, L. y E. Zeiger. 2006. Plant physiology. $4^{\text {th }}$ ed. Sinauer Associates Publishers, Sunderland, MA.

Talón, M. 2000. Giberelinas. pp. 301-317. En: AzcónBieto, J. y M. Talón (eds.). Fundamentos en fisiología vegetal. McGraw-Hill, Madrid.

Taylorson, R.B. y S.B. Hendricks. 1977. Dormancy in seeds. Annu. Rev. Plant Physiol. 28(1), 331-354.

Weaver, R.J. 1996. Reguladores del crecimiento de las plantas de la agricultura. Editorial Trillas, México.

Wilches-Rojas, F. J.G. Álvarez-Herrera y H.E. Balaguera López. 2008. Tamaños de alvéolo y diferentes láminas de riego en obtención de plántulas de tomate. Rev. UDCA Actual. Divulg. Cient. 11(2), 141-151. 